\title{
Exploring Pen and Paper Interaction with High-Resolution Wall Displays
}

\author{
Nadir Weibel, Anne Marie Piper, James D. Hollan \\ Distributed Cognition and Human Computer Interaction Lab \\ Department of Cognitive Science, University of California, San Diego \\ 9500 Gilman Dr., La Jolla, CA 92093-0515, USA \\ \{weibel, apiper, hollan\}@ucsd.edu
}

\begin{abstract}
We introduce HIPerPaper, a novel digital pen and paper interface that enables natural interaction with a 31.8 by 7.5 foot tiled wall display of $268,720,000$ pixels. HIPerPaper provides a flexible, portable, and inexpensive medium for interacting with large high-resolution wall displays. While the size and resolution of such displays allow visualization of data sets of a scale not previously possible, mechanisms for interacting with wall displays remain challenging. HIPerPaper enables multiple concurrent users to select, move, scale, and rotate objects on a high-dimension wall display.
\end{abstract}

ACM Classification: H.5.2 [Information Interfaces and Presentation]: User Interfaces - Input devices and strategies

General terms: Design, Human Factors

Author Keywords: Wall Display, Digital Pen, Paper INTRODUCTION

The decreasing cost of displays and growing computational power of graphics processing units (GPUs) are enabling visualization and analysis of increasingly large multidimensional data sets on tiled wall displays. The scale of these data sets means they cannot be viewed or easily manipulated on desktop or simple projection displays. High-resolution wall displays promise to be particularly useful for information visualization, allowing visual detection of patterns in data that would otherwise be impossible to see on current desktop display technology.

A range of interaction techniques for large wall displays have been explored in the past $[2,1,10,9,4]$. One promising approach we are exploring is to use pen and paper, another high-resolution medium, as the main interface for controlling an experimental tiled wall composed of seventy 30 " displays. Prior work involving pen-based interaction with large displays $[5,3,6]$ focuses on direct pen/display interaction and does not investigate the potential of using pen and paper as an indirect mechanism to control large high-resolution displays. Pen and paper interaction with a large wall display is promising for several reasons. Paper is lightweight and flexible and it allows for myriad types of interfaces, from simple commands to regions providing special functions such as zooming or panning. Paper can be customized easily to suit various tasks and interaction needs, then discarded after use. Paper is mobile, meaning that users can freely navigate in the space in front of the display.

Copyright is held by the owner/author(s).

UIST'10, October 3-6, 2010, New York, New York, USA.

ACM 978-1-4503-0271-5/10/10

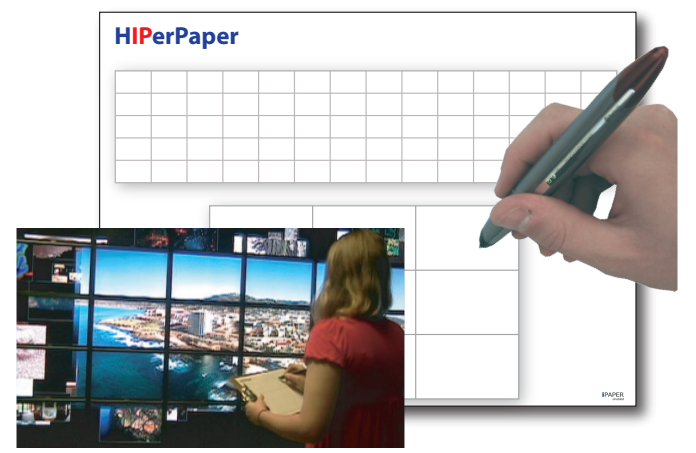

Figure 1: HIPerPaper Interface

The HIPerSpace Wall ${ }^{1}$ is a 31.8 foot wide by 7.5 foot tall wall display composed of seventy tiled 30" Dell LCD displays with a resolution of $35,840 \times 8,000$ pixels $(286,720,000$ pixels total). Observations of users interacting with the HIPerSpace wall reveal three key challenges: (1) Viewing information on and interacting with the wall involves multiple people working in coordination; (2) The wall display is designed to support interaction with large multidimensional data sets, but setup time and lack of natural mechanisms for manipulating data results are an issue; and (3) The size and physical layout encourages movement and interaction throughout the room, necessitating a mobile interface for interaction.

\section{HIPERPAPER}

HIPerPaper is a new interface that provides pen and paper interaction with large wall displays. It addresses the above concerns involving multiuser interaction, the need for simple ways of interacting with data visualizations, and the potential to support group interactions exploiting the workspace surrounding the display. The interface is based on Anoto technology and the iPaper framework [8]. It exploits a novel architecture for handling multi-user, multi-device, multi-channel and multi-modal interactions. The main interface (see Fig. 1) is a scaled version of the HIPerSpace wall printed on paper. Multiple users can interact with the wall using multiple paper-based interfaces and digital pens. Users may interact with the paper interface using either a traditional or non-marking pen tip. The upper region of the main paper interface supports coarse navigation of the wall display. The lower region provides multiscale access to a particular part of the display (i.e., an area the user is interacting with on the large overview), allowing fine positioning. The design goal is to encourage that the main focus of attention is on the high-resolution wall display.

\footnotetext{
${ }^{1}$ http://vis.ucsd.edu/projects/hiperspace/
} 


\section{Paper-based interactions}

We use basic $(\mathrm{x}, \mathrm{y})$ position tracking of the pen to move a pointer on the display in the default navigation mode. By moving a pen with a non-marking tip on the paper-wall, users can control a cursor moving and dragging objects on the display (Fig. 2, left). Scaling is one of the most valuable functions on high-resolution wall displays. HIPerPaper introduces a general scaling interface based on a circular interaction with the paper interface [7]. Similar to scrolling through music on an Apple iPod, users can scale an object by first selecting it and then moving then pen circularly adjusting their path and speed of movement for fine or coarse scaling (Fig. 2, right).
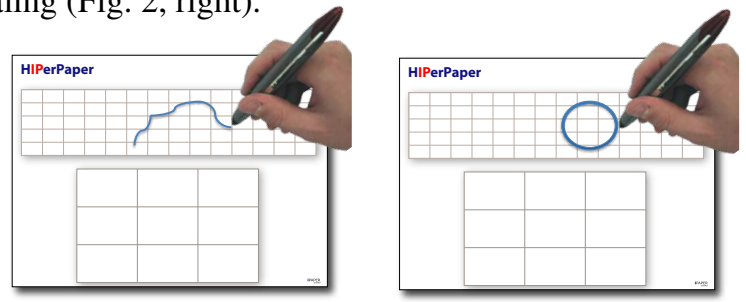

Figure 2: Interacting with the HIPerPaper interface

Viewing information and interacting with the HIPerSpace wall almost always involves multiple people working in collaboration. Users can interact with the wall using multiple paper-based interfaces and digital pens while exploiting the supported interactions for collaboration.

In addition to the main paper interface, the current system incorporates a set of paper-based buttons and widgets (Fig. 3). Touching or moving the pen tip on these widgets issues commands to the application running on the wall. The paper buttons and widgets can be printed directly on the main paper interface, printed on separate papers sheets, or deployed in the form of removable stickers to be placed in convenient locations such as on a clipboard, a table, or even on the face of a watch or other body location.

Finally, the information derived from the timestamped $(\mathrm{x}, \mathrm{y})$ position of the digital pen enables multiple forms of interaction. For example, to access a close-up view (lower part of Fig. 1), we exploit velocity in combination with a simple linear gesture. Users can move the close-up view to a different section of the wall display with a simple horizontal or vertical panning gesture in the desired direction (differentiated by the pen's velocity). The graphic component driving all HIPerSpace applications supports an overlay layer that we plan to exploit as an interactive feedback mechanism for showing the particular region of the display that is currently controlled by the close-up view.

\section{FUTURE WORK: A LABORATORY FOR INTERACTIONS}

The central research challenge we address is twofold: to identify pen-based interaction techniques for large wall displays that are effective and natural for specific contexts, and to design an infrastructure that enables their implementation, composition, and exploration. HIPerPaper supports rapid development, deployment, and evaluation of new prototype interactions.

One advantage of paper interfaces is that they are extremely easy to define and deploy (i.e., print). Interactive paper allows a wide range of interfaces in terms of their layout and presentation but also in terms of how interaction is imple-

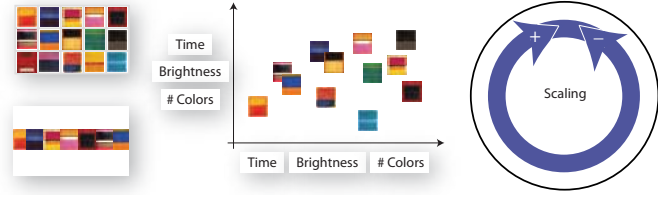

Figure 3: HIPerPaper widgets

mented. HIPerPaper enables exploration of widgets that enable direct access to data visualizations within various contexts. More general paper-based tools that enable interactions across applications can be introduced.

HIPerPaper not only enables flexibility in terms of widgets but also exploits the portable nature of paper, particularly valuable when interacting with a large wall display. We plan to deploy universal paper-based controls on desk surfaces throughout the room for use by any user interacting with any of the applications running on the HIPerSpace Wall.

While individual interaction techniques can be beneficial for certain tasks, a single technique is unlikely to be appropriate for all activities and contexts. The wall display we examine is a novel experimental setting and it is important to be able to explore multiple ways of interacting. We expect use practices to co-evolve with the interaction mechanisms we make available to users. Creating a flexible and extensible architecture that supports a variety of input devices and interaction techniques is one long-term objective of our research.

\section{ACKNOWLEDGMENTS}

This work is funded by NSF grant 0729013 . We thank KaiUwe Dörr, So Yamaoka, Amanda Lazar, Arvind Satyanarayan, Reid Oda and Amanda Legge for the precious help.

\section{REFERENCES}

1. R. Ball, C. North, and D. A. Bowman. Move to improve: promoting physical navigation to increase user performance with large displays. In Proc. CHI '07, pages 191-200, 2007.

2. R. Ballagas, M. Rohs, and J. G. Sheridan. Sweep and point and shoot: phonecam-based interactions for large public displays. In Proc. CHI '05, pages 1200-1203, 2005.

3. P. Baudisch, E. Cutrell, D. Robbins, et al. Drag-and-pop and drag-and-pick: Techniques for accessing remote screen content on touch- and pen-operated systems. In Proc. Interact '03, pages $57-64,2003$.

4. A. Bornik, R. Beichel, E. Kruijff, B. Reitinger, and D. Schmalstieg. A hybrid user interface for manipulation of volumetric medical data. In Proc. 3DUI '06, pages 29-36, 2006.

5. F. Guimbretière, M. Stone, and T. Winograd. Fluid interaction with high-resolution wall-size displays. In Proc. UIST '01, pages 21-30, 2001.

6. M. Haller, J. Leitner, T. Seifried, et al. The nice discussion room: Integrating paper and digital media to support co-located group meetings. In Proc. CHI '10, pages 609-618, 2010.

7. S. Malacria, E. Lecolinet, and Y. Guiard. Clutch-free panning and integrated pan-zoom control on touch-sensitive surfaces: the cyclostar approach. In Proc. CHI '10, pages 2615-2624, 2010.

8. M. C. Norrie, B. Signer, and N. Weibel. General Framework for the Rapid Development of Interactive Paper Applications. In Proc. CoPADD 2006, pages 9-12, 2006.

9. J. Sanneblad and L. E. Holmquist. Ubiquitous graphics: combining hand-held and wall-size displays to interact with large images. In Proc. AVI '06, pages 373-377, 2006.

10. E. Tse, M. Hancock, and S. Greenberg. Speech-filtered bubble ray: improving target acquisition on display walls. In Proc. ICMI'07, pages 307-314, 2007. 